\title{
Electron tunneling through alkanedithiol molecules
}

\author{
R. C. Hoft, J. Liu, M. B. Cortie, and M. J. Ford, \\ Institute for Nanoscale Technology, University of Technology Sydney, P. O. Box 123, Broadway, \\ NSW 2007, Australia
}

\begin{abstract}
We report on first principles calculations of the tunneling current across $n$-alkanedithiol molecules $(n=4,6,8,10,12)$ sandwiched between two $\mathrm{Au}\{111\}$ electrodes. The conductance drops exponentially with increased chain length with decay parameter $\beta_{n}=0.9$. The results are compared with scanning tunneling microscopy measurements on decanedithiol and with other $n$-alkanedithiol $(n=6,8,10)$ results in the literature. The theoretical results are found to be an order of magnitude larger than experimental values but follow the same trend. However, two additional, more realistic, geometries are modeled by changing the bond type and by combining the first-principles results with a Wentzel-KramerBrillouin (WKB) expression for tunneling across the air gap that is invariably present during scanning tunneling microscopy (STM) measurements. These results are more compatible with the experimental data.
\end{abstract}

Keywords: Electron tunneling, scanning tunneling spectroscopy, molecular electronics, self-assembled monolayer, molecular conductance

\section{INTRODUCTION}

Tunneling of electrons between metal electrodes in the presence of adsorbed molecules has attracted considerable interest in recent years ${ }^{1}$. It is a problem of significant scientific interest and is claimed to have a number of potential applications, such as the creation of molecular devices. An understanding of the electron transfer across monolayercoated electrodes in existing and future biomedical devices is also important, particularly as the conductivity and capacitance of such interfaces may be affected by adsorbed proteins and other adventitious bio-molecules.

Alkanedithiol molecules are believed to form stable monolayers on gold $\{111\}$ surfaces facilitated by a strong goldsulfur pseudo-covalent bond. ${ }^{2}$ STM measurements and other studies of electron transport through conjugated molecules are often performed with the conjugated molecules embedded in a matrix of an alkanedithiol. ${ }^{3-6}$ The alkane chains are believed to be more insulating due to the localized nature of their orbitals, as opposed to the more conductive conjugated molecules.

There have also been several reports of first-principles calculations of tunneling currents through aromatic molecules such as dithiol benzene ${ }^{7}$, and recent state-of-the-art experiments have measured the conductance of these and $n$ alkanedithiol monolayers for $n=6,8,10^{8}$. However, we are not aware of any systematic, first principles calculations of the tunneling currents through a series of $n$-alkanedithiol molecules, where $n=4,6,8,10,12$. This is a point that we shall address here.

\section{METHOD AND RESULTS}

Geometry optimizations of decanedithiol on a $\mathrm{Au}\{111\}$ surface were performed using the density functional code, SIESTA $^{9}$. The surface is approximated by 4 layers of $3 \times 3$ gold atoms and the other parameters and method are described in our earlier work ${ }^{10}$. We assume a bonding geometry where the hydrogen atom bonded to the sulfur is removed and the sulfur thus forms a strong pseudo-covalent bond to the surface, which we shall refer to as a thiolate bond. The optimal 
binding site was found to be between the bridge and fcc-hollow sites with the sulfur atom at a height of $2.1 \AA$ above the gold surface. The optimal tilt angle was found as $18^{\circ}$ to the surface normal. The binding of the other alkanedithiols to the $\mathrm{Au}\{111\}$ surface was assumed to be the same and no further optimizations were performed. To obtain the electrodemolecule-electrode geometries, the tilt angles had to be adjusted slightly in order to place the sulfur atom on either end of the molecule in equivalent surface sites on the opposite electrodes. Over the range of angles used from $14^{\circ}$ to $24^{\circ}$ the interaction energy varies by less than $0.4 \mathrm{kcal} / \mathrm{mol}$ and we therefore feel confident that this will not affect the results significantly. The current between electrodes due to an applied bias is calculated with the TRANSIESTA ${ }^{11}$ package. This first-principles code calculates the electronic structure in the spirit of the SIESTA method and uses non-equilibrium Green's functions to find the transmission spectrum and hence current for a given inter-electrode bias.

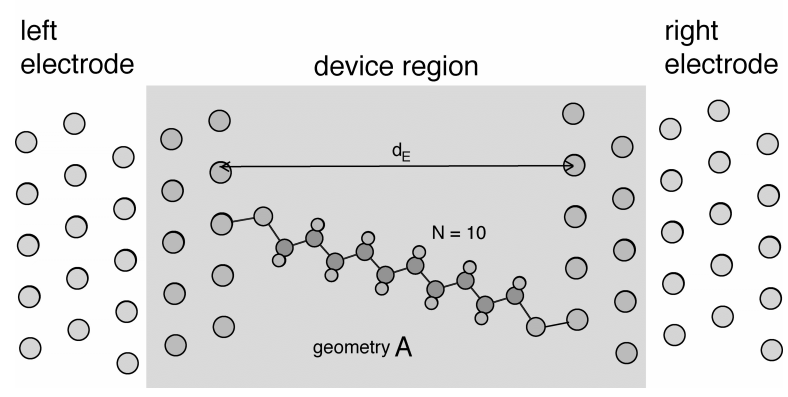

Figure 1: Conventional Au $\{111\}-\mathrm{C} 10-\mathrm{Au}\{111\}$ computational setup (geometry A). Each layer of $\mathrm{Au}\{111\}$ is represented by $3 \times 3$ $\mathrm{Au}$ atoms. The device region consists of the molecule sandwiched between two layers of gold on either side. Semi-infinite electrodes extend on either side of the device region in the $z$-direction. The system is repeated periodically in the $x$ - and $y$-directions for the purposes of the computation.

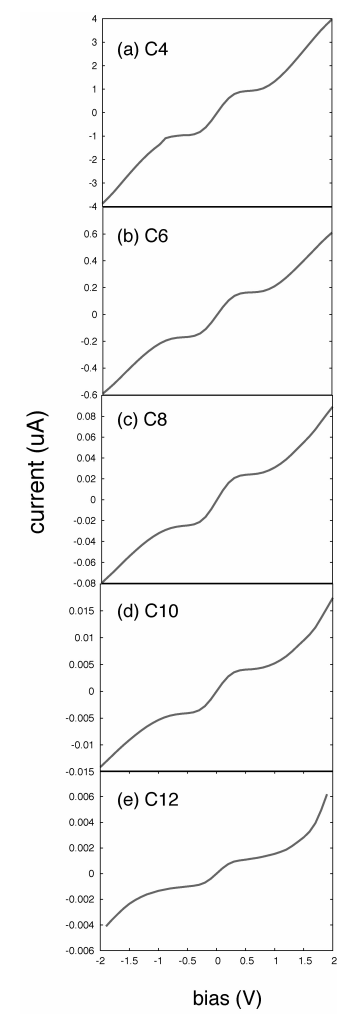

Figure 2: First-principles current-voltage curves for $n$-alkanedithiol, $n=$ (a) 4, (b) 6, (c) 8, (d) 10, (e) 12 (geometry A) 
The two-probe geometry for decanedithiol is illustrated in Fig. 1. This is the conventional geometry used in theoretical calculations of tunneling current through a molecule where both ends of the molecule form a thiolate bond with the respective electrodes and we name it geometry A. The molecule together with two layers of Au $\{111\}$ is used as the device region, attached to a semi-infinite electrode on either side. The calculations were performed for bias values between $-2.0 \mathrm{~V}$ and $2.0 \mathrm{~V}$ in steps of $0.1 \mathrm{~V}$ in order to find the $i(V)$ curve for each molecule shown in Fig. 2.

Due to the localized nature of the $\sigma$-orbitals on the alkane chains, the transport process is expected to be dominated by tunneling and hence the conductance should decrease exponentially with chain length. Fig. 3 shows the $0 \mathrm{~V}$-conductance plotted against (a) the number of carbon atoms in the chain $n$ and (b) the inter-electrode distance $d_{E}$.

We thus identify two decay parameters, $\beta_{n}=0.90$ by fitting

$$
G=A_{n} e^{-\beta_{n} n}
$$

to the $(n, G)$ data in Fig. 3 (a) and $\beta=0.72$ by fitting

$$
G=A e^{-\beta d_{E}}
$$

to the $\left(d_{E}, G\right)$ data in Fig. 3 (b) with $G$ the conductance, $n$ the number of carbon atoms in the chain and $d_{E}$ the interelectrode distance. This is in good agreement with the experimental values, $\beta_{n}=1.0$ and $\beta=0.9$ from Xu et al. ${ }^{8}$, plotted on Fig. 3. On Fig. 3 (b) we also show our theoretical values (which will be reported more thoroughly elsewhere ${ }^{12}$ ) as well as experimental values from Xiao et $a l .{ }^{13}$ for benzenedithiol (DTB) and 1,4 phenylenedimethanethiol (XYL). Since these two molecules contain a phenyl ring with delocalized $\pi$-orbitals, they are believed to be more conductive than the alkane chains. This is in agreement with our theoretical values where their conductivities lie above the line fitted to the $n$-alkanedithiol data. However the results from Xiao et al. ${ }^{13}$ lie below the line fitted to the $n$-alkanedithiol results from Xu et $a l^{8}{ }^{8}$ This is surprising since the two sets of data were obtained by the same group using the same technique.

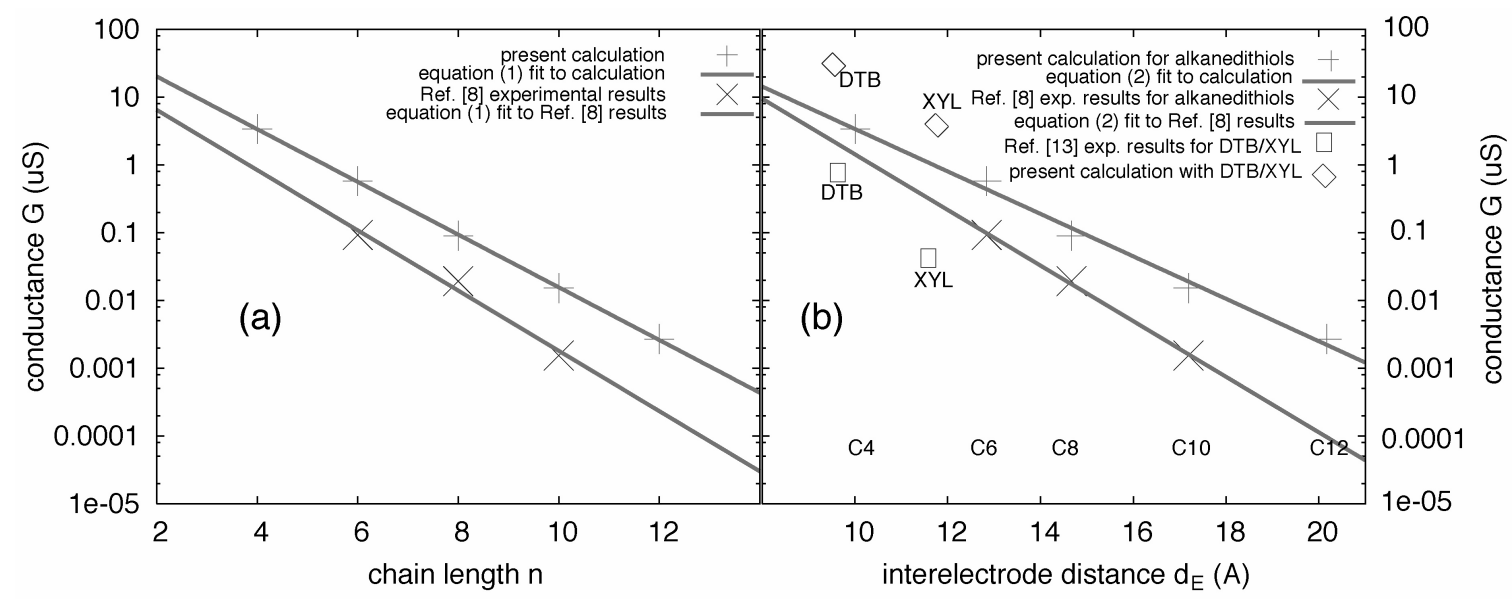

Figure 3: 0V-conductance of $n$-alkane chains plotted against (a) the number of carbon atoms in the chain $n$ and (b) the interelectrode distance $d_{E}$.

Another point to note is that the experimental results on Fig. 3 (b) are consistently about an order of magnitude smaller than our theoretical values. This disagreement between experiment and theory has been noted by other authors ${ }^{7,14}$. Although problems with the application of density functional theory have been pointed out by various authors ${ }^{15-18}$, we believe that an important factor is the difference between the perfect bonding geometry (A) depicted in theoretical 
calculations and the actual experimental geometry which is not known. Accordingly, we model two alternate geometries for the C10 molecule:

(B) A gold nanoparticle absorbed onto a monolayer with a gap distance $d_{\text {gap }}$ between the nanoparticle and a probing tip, the bias being applied between tip and substrate (Fig. 4 (a)).

(C) One end of the molecule thiolate bonded to the bottom electrode as before and the other end forming a weaker bond to the top electrode where the hydrogen atom bonded to the sulfur atom is not removed (Fig. 4 (b)). We refer to this as a thiol bond. We label the sulfur to surface distance of this thiol bond $d_{S}$.
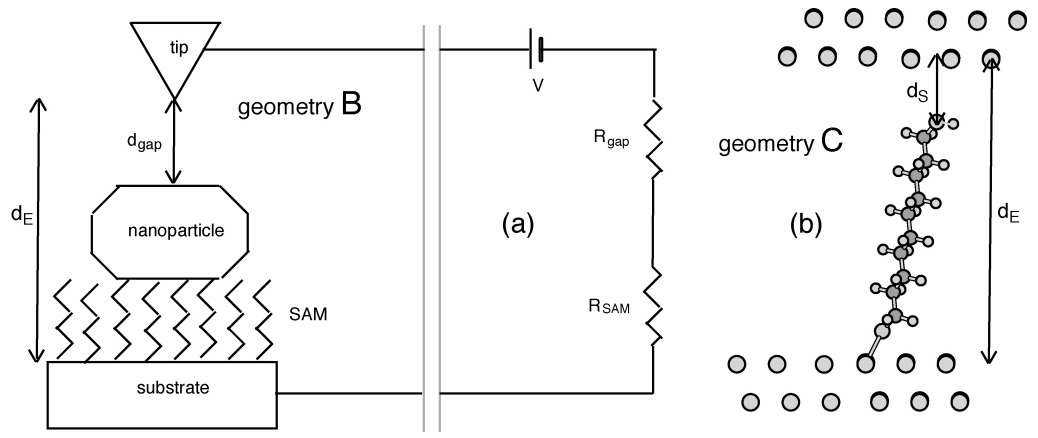

Figure 4: (a) Model of geometry B. The bias is applied between STM tip and substrate. A monolayer is adsorbed onto the substrate and a gold nanoparticle is absorbed onto the monolayer. A gap of size $d_{\text {gap }}$ is present between the nanoparticle and tip. (b) Geometry $\mathrm{C}$ : the molecule is thiolate bonded to the bottom electrode and thiol bonded to the top electrode (see text for definition of bond types).

The thiol bond has sulfur to surface distance $d_{S}$.

Fig. 4 (a) shows geometry B. The substrate-molecule-nanoparticle geometry is assumed to be the same as in geometry A and for the $i(V)$ characteristic of this subsystem we use the first-principles results as before. We model tunneling through the gap with the WKB approximation for the transmission function $T(E)$ between electrodes

$$
T\left(E, V, d_{g a p}\right)=e^{-\frac{2 \sqrt{2}}{\hbar} \int_{0}^{l_{g a p}} \sqrt{m(U(x)-E)} d x}
$$

where $m$ is the electron mass, $d_{\text {gap }}$ is the width of the barrier and $U(x)$ is the potential function across the barrier. The current is then found from the Landauer formalism as

$$
i\left(V, d_{\text {gap }}\right)=\frac{2 e}{h} \int_{-\infty}^{\infty} T\left(E, V, d_{g a p}\right) \rho_{t}(E-e V) \rho_{p}(E)[f(E)-f(E-e V)]
$$

where $\rho_{\text {tip }}$ and $\rho_{\text {particle }}$ are the local densities of states of the tip and particle, $V=V_{\text {particle }}-V_{\text {tip }}$ with the convention that the tip is grounded and $2 e / h \approx 77 \mu \mathrm{S}$ is the contact conductance ${ }^{19}$. We use the zero temperature approximation and assume a constant density of states to find the simplified relation

$$
i\left(V, d_{\text {gap }}\right)=\frac{2 e}{h} \int_{0}^{e V} e^{-\frac{2 \sqrt{2}}{\hbar} \int_{0}^{d_{g a p}} \sqrt{m(U(x)-E} d x} d E
$$

We use a trapezoidal barrier with barrier height given by the work function of gold $\phi \approx 5 \mathrm{eV}$ and modified by a sinusoidal function which approximately accounts for image charging ${ }^{20}$ 


$$
U(x)=e V\left(1-\frac{x}{d_{g a p}}\right)+\phi\left[\sin \left(\frac{\pi x}{d_{g a p}}\right)\right]^{\alpha}
$$

with the dimensionless parameter $\alpha=0.2$.

With the tip-nanoparticle $i\left(V, d_{\text {gap }}\right)$ function thus given and the nanoparticle-substrate $i(V)$ data calculated from firstprinciples, we can obtain the tip-substrate $i(V)$ and $i\left(d_{\text {gap }}\right)$ characteristics. In Fig. 5 we plot the $i\left(d_{\text {gap }}\right)$ function for $n$ alkanedithiol $(n=4,6,8,10,12)$ at a fixed bias of $0.3 \mathrm{~V}$. At gap distances above $0.6 \mathrm{~nm}$, only the gap would be probed and the particular molecule does not influence the results.

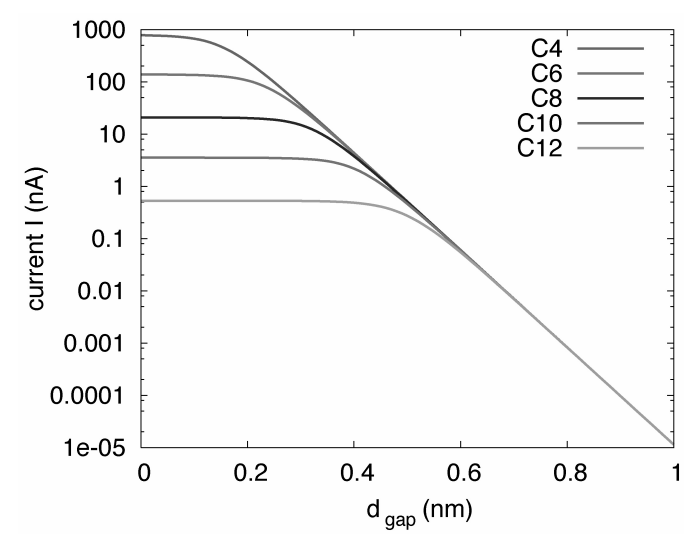

Figure 5: $i\left(d_{\text {gap }}\right)$ results for geometry B (see Fig. 4 (a)). The first-principles current through $n$-alkanedithiol $(n=4,6,8,10,12)$ is convoluted with the WKB current through a gap between an adsorbed Au nanoparticle and a probing tip as a function of the gap distance $d_{\text {gap }}$ at a fixed tip-substrate bias of $0.3 \mathrm{~V}$.

We have also performed STM experiments with this geometry (B) using a SAM of decanedithiol. The SAM had been assembled onto a clean $\mathrm{Au}\{111\}$ substrate from an ethanolic solution. The results of five successive runs of the experiment are shown in Fig. 6.

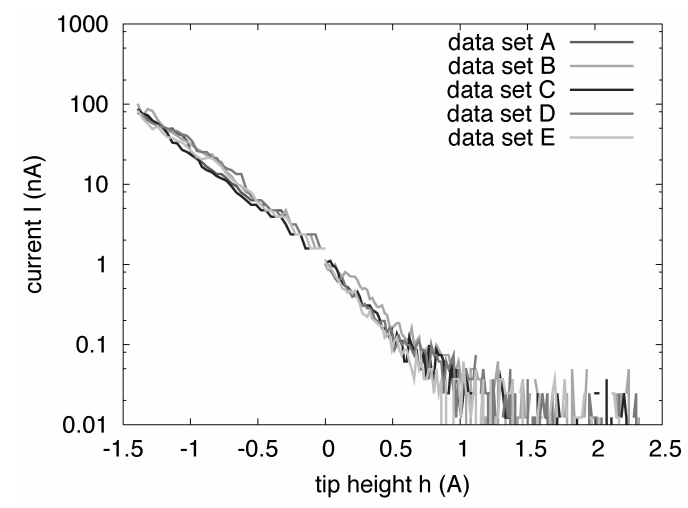

Figure 6: Tunneling current values at a fixed bias of $0.3 \mathrm{~V}$ using a scanning tunneling microscope. The tip was started at an unknown height above the substrate indicated as $h=0$. It was then pulled off further or approached closer to the surface and current readings taken at these relative heights. The discontinuity in the data at $h=0$ indicates that the starting height was not identical for the pulling off and approaching runs. 
The diameter of the nanoparticles is $\sim 15 \mathrm{~nm}$. If we assume the usual $\sqrt{3} \mathrm{x} \sqrt{3} \mathrm{R} 30^{\circ}$ symmetry for the monolayer, then the intermolecular distance is $\sim 0.5 \mathrm{~nm}$. Thus we calculate roughly 700 molecules per nanoparticle. Dividing the largest experimental current of about $100 \mathrm{nA}$ (see Fig. 6) equally amongst these 700 molecules, gives a current of $\sim 0.15 \mathrm{nA}$ per molecule. Relating this to the calculated C10 data of Fig. 5, gives a nanoparticle-tip distance $d_{\text {gap }}$ of just below $0.6 \mathrm{~nm}$, inside the regime where we are probing only the $i(V)$ characteristic of the air gap. The shape of our experimental $i\left(d_{\text {gap }}\right)$ data thus compares well with the shape of the theoretical $i\left(d_{\text {gap }}\right)$ data if we assume $d_{\text {gap }}>0.6 \mathrm{~nm}$.

To model geometry $\mathrm{C}$, we used the first-principles code TRANSIESTA exclusively. We have previously calculated the bonding geometry of a sulfur thiol bonded to a $\mathrm{Au}\{111\}$ surface with the SIESTA software package ${ }^{12}$ and found the sulfur-surface distance $d_{S}=2.39 \AA$ and the binding site between the on-top and hcp-hollow sites. We use this geometry to place the $\mathrm{C} 10$ molecule between two $\mathrm{Au}\{111\}$ electrodes, thiolate and thiol bonded to the bottom and top surfaces respectively. We calculate the current at a fixed bias of $0.3 \mathrm{~V}$ and the conductance at $0 \mathrm{~V}$ as the right electrode is pulled away from the thiol-bonded sulfur to distances of $d_{S}=3.0 \AA$ and $d_{S}=4.0 \AA$ to model the effect of a gap between molecule and electrode. Fig. 7 shows the current and conductance decay exponentially with sulfur-surface distance $d_{S}$. The $\beta$ decay parameter for the $0 \mathrm{~V}$-conductance in this case is $\beta=0.85$. The $0.3 \mathrm{~V}$ current at the equilibrium sulfursurface distance $d_{S}=2.39 \AA$ is $1.04 \mathrm{nA}$. The experimental current at an applied bias of $0.3 \mathrm{~V}$ is $\sim 0.5 \mathrm{nA}$ as measured by $\mathrm{Xu}$ et $a l^{8}{ }^{8}$. Geometry $\mathrm{C}$ gives a current in much better agreement with this experimental value compared with our theoretical result with geometry A where $i(0.3 \mathrm{~V})=3.5 \mathrm{nA}$. The comparison to experiment becomes even better if $d_{S}$ is increased above the equilibrium distance. Again this shows how discrepancies between theoretical and experimental results can be ascribed to the uncertainty in geometry.

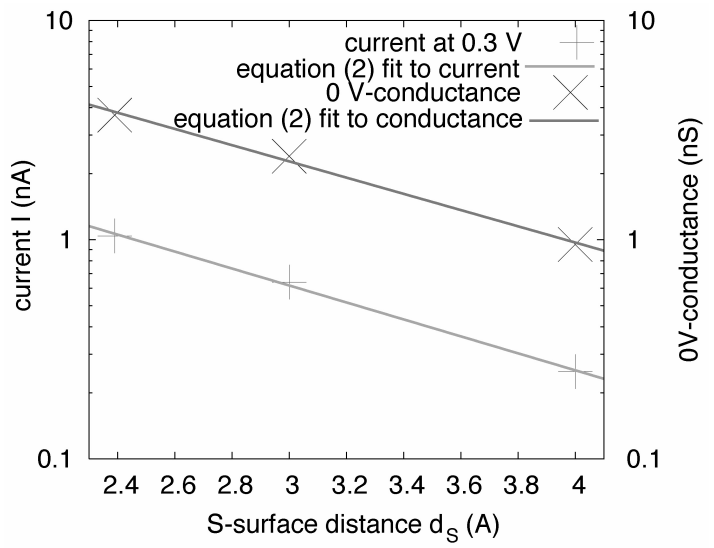

Figure 7: The first principles current at $0.3 \mathrm{~V}$ bias and conductance at $0 \mathrm{~V}$ bias for geometry $\mathrm{C}$ : a $\mathrm{C} 10$ molecule thiolate bonded to the bottom electrode and thiol bonded to the top electrode as the top electrode is pulled away with sulfur-surface distance $d_{S}$ on the x-axis.

\section{CONCLUSION}

We performed first principles calculations of the current through a series of alkane chains ( $n$-alkanedithiol with $n=$ 4,6,8,10 and 12 carbon atoms) adsorbed between two $\mathrm{Au}\{111\}$ electrodes. The conductance was found to decay exponentially with decay parameters similar to those found in recent state-of-the-art experiments. Our theoretical results for two phenyl ring based molecules, DTB and XYL, indicate that these conjugated molecules are indeed more conductive than the alkane chains.

As has been found in other studies, the initial theoretical results are about an order of magnitude larger than experimental values. In addition to this conventional theoretical geometry (A), we modeled two additional geometries: (B) a gold nanoparticle adsorbed on a SAM with an additional gap between gold nanoparticle and top electrode and (C) a different bond type between the top end of the molecule and the top electrode. We show that these more realistic geometries bring the theoretical results in closer agreement with experiment. 


\section{ACKNOWLEDGEMENTS}

This work was supported by the Australian Research Council and University of Technology, Sydney. Computing facilities were accessed through the merit allocation schemes of the National Facility at the Australian Partnership for Advanced Computing, and the Australian Centre for Advanced Computing and Communications in New South Wales.

\section{REFERENCES}

1. D. K. James and J. M. Tour, Chemistry of Materials 164423 (2004).

2. J. C. Love, L. A. Estroff, J. K. Kriebel, R. G. Nuzzo et al., Chemical Reviews 1051103 (2005).

3. K. Moth-Poulsen, L. Patrone, N. Stuhr-Hansen, J. B. Christensen et al., Nano Letters 5783 (2005).

4. L. A. Bumm, J. J. Arnold, M. T. Cygan, T. D. Dunbar et al., Science 2711705 (1996).

5. G. Leatherman, E. N. Durantini, D. Gust, T. A. Moore et al., J Phys Chem B 1034006 (1999).

6. Z. J. Donhauser, B. A. Mantooth, K. F. Kelly, L. A. Bumm et al., Science 2922303 (2001).

7. K. Stokbro, J. Taylor, M. Brandbyge, J. L. Mozos et al., Computational Materials Science 27151 (2003).

8. $\quad$ B. Q. Xu and N. J. J. Tao, Science 3011221 (2003).

9. J. M. Soler, E. Artacho, J. D. Gale, A. Garcia et al., J Phys: Condens Mat 142745 (2002).

10. M. J. Ford, R. C. Hoft, and A. McDonagh, J Phys Chem B 10920387 (2005).

11. M. Brandbyge, K. Stokbro, J. Taylor, J. L. Mozos et al., Phys Rev B 67 (2003).

12. R. C. Hoft, M. Ford, and M. B. Cortie, (in preparation) (2005).

13. X. Y. Xiao, B. Q. Xu, and N. J. Tao, Nano Letters 4267 (2004).

14. M. Di Ventra, S. T. Pantelides, and N. D. Lang, Phys Rev Lett 84979 (2000).

15. G. C. Solomon, J. R. Reimers, and N. S. Hush, J Chem Phys 1216615 (2004).

16. P. S. Krstic, D. J. Dean, X. G. Zhang, D. Keffer et al., Computational Materials Science 28321 (2003).

17. F. Evers, F. Weigend, and M. Koentopp, Phys Rev B 69 (2004).

18. A. Bilic, J. R. Reimers, and N. S. Hush, J Chem Phys 122 (2005).

19. S. Datta, Electronic transport in mesoscopic systems. (Cambridge University Press, 1995).

20. M. B. Cortie, M. H. Zareie, S. R. Ekanayake, and M. J. Ford, IEEE Transactions on Nanotechnology 4406 (2005). 